PROCEEDINGS OF THE

AMERICAN MATHEMATICAL SOCIETY

Volume 139, Number 6, June 2011, Pages 1989-1992

S 0002-9939(2010)10777-3

Article electronically published on November 15, 2010

\title{
A NOTE ON PROJECTIVE NORMALITY
}

\author{
HUAH CHU, SHOU-JEN HU, AND MING-CHANG KANG
}

(Communicated by Ted Chinburg)

\begin{abstract}
Let $G$ be any finite group, $G \rightarrow G L(V)$ be a representation of $G$, where $V$ is a finite-dimensional vector space over an algebraically closed field $k$. Theorem. Assume that either char $k=0$ or char $k=p>0$ with $p \nmid|G|$. Then the quotient variety $\mathbb{P}(V) / G$ is projectively normal with respect to the line bundle $\mathcal{L}$, where $\mathcal{L}$ is the descent of $\mathcal{O}(1)^{\otimes m}$ from $\mathbb{P}(V)$ with $m=|G|$ !. This partially solves a question raised in the paper of Kannan, Pattanayak and Sardar [Proc. Amer. Math. Soc. 137 (2009), 863-867].
\end{abstract}

\section{INTRODUCTION}

Let $k$ be an algebraically closed field, $G$ be a finite group, $G \rightarrow G L(V)$ be a representation of $G$ to $G L(V)$, where $V$ is a finite-dimensional vector space over $k$. Then $G$ acts on $V$ and $\mathbb{P}(V)$ (which is the projective space $\mathbb{P}^{r-1}$ if $\operatorname{dim}_{k} V=r$ ).

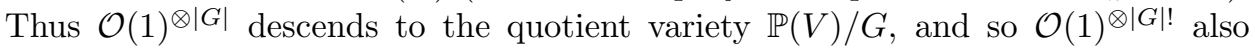
descends to the quotient variety (see $[\mathrm{KKV}]$ for details). In [KPS, the projective normality of $\mathbb{P}(V) / G$ with respect to the descent of $\mathcal{O}(1)^{\otimes|G|}$ is considered. The following result is proved there.

Theorem 1.1 (Kannan, Pattanayak and Sardar [KPS, Theorem 3.1]). Let $\mathcal{L}$ be the line bundle on $\mathbb{P}(V) / G$ which is the descent of $\mathcal{O}(1)^{\otimes m}$ from $\mathbb{P}(V)$, where $m=|G|$. Assume that either char $k=0$ or char $k=p>0$ with $p \nmid|G|$. If $G$ is a solvable group or a pseudo-reflection group, then $\mathbb{P}(V) / G$ is projectively normal with respect to $\mathcal{L}$.

The reader is referred to $\mathrm{KP}$ for progress made on this question for Weyl group representations. What we will prove in this article is the following theorem, which holds for any finite group.

Theorem 1.2. For any finite group $G$, let $\mathcal{L}$ be the line bundle on $\mathbb{P}(V) / G$ which is the descent of $\mathcal{O}^{\otimes m}$ from $\mathbb{P}(V)$, where $m=|G|$ !. Assume that either char $k=0$ or char $k=p>0$ with $p \nmid|G|$. Then $\mathbb{P}(V) / G$ is projectively normal with respect to $\mathcal{L}$.

Before stating a key lemma for proving Theorem [1.2, we give the definition of the "restricted product".

Received by the editors December 6, 2009 and, in revised form, June 4, 2010.

2010 Mathematics Subject Classification. Primary 13A02, 13A50, 14Lxx.

Key words and phrases. Projectively normal, rings of invariants, graded algebras.

(C)2010 American Mathematical Society Reverts to public domain 28 years from publication 
Definition 1.3. Let $a_{1}, \ldots, a_{n}$ be positive integers (it is allowed that $a_{i}=a_{j}$ for some $i \neq j$ ). The usual product is simply $a_{1} a_{2} \cdots a_{n}$ (for short, $\prod_{i} a_{i}$ ). We will define $\prod_{i}^{\prime} a_{i}$, the restricted product, as $\prod_{i}^{\prime} a_{i}=\prod_{1 \leq j \leq e} b_{j}$ if $\left\{a_{1}, a_{2}, \ldots, a_{n}\right\}=$ $\left\{b_{1}, b_{2}, \ldots, b_{e}\right\}$, where $b_{i} \neq b_{j}$ if $i \neq j$.

Lemma 1.4. Let $k$ be a field, $A=A_{0} \oplus A_{1} \oplus \cdots \oplus A_{l} \oplus \cdots$ be a commutative graded algebra, where $A_{0}=k$ and $A_{l}$ is the k-vector space of homogeneous elements of degree $l$ in $A$. Let $A=k\left[f_{1}, \ldots, f_{n}\right]$ for some homogeneous elements $f_{1}, \ldots, f_{n}$ with $\operatorname{deg} f_{i}=a_{i} \geq 1$. Define $m=\prod_{i}^{\prime} a_{i}$ to be the restricted product of $a_{1}, a_{2}, \ldots, a_{n}$. Then $k\left[A_{m}\right]=A_{0} \oplus A_{m} \oplus A_{2 m} \oplus \cdots \oplus A_{d m} \oplus \cdots$, where $k\left[A_{m}\right]=k\left[g_{1}, \ldots, g_{r}\right]$ if $A_{m}=\sum_{1 \leq j \leq r} k \cdot g_{j}$.

We remark that the above lemma was known to Zariski when $A$ is the integral closure of some graded $k$-algebra $B=B_{0} \oplus B_{1} \oplus \cdots \oplus B_{l} \oplus \cdots$ (with $B_{0}=k$ ) such that $\operatorname{Proj}(B)$ is a normal variety and $B$ is generated by $B_{1}$ over $k$ (see [Za]; $\mathrm{La}$, Proposition 11, p. 141; Ha, Exercise 5.14, p. 126).

\section{ProOF}

We will denote by $\mathbb{N}$ the set of all positive integers, and by $\mathbb{N}_{\geq 0}$ the set $\mathbb{N} \cup\{0\}$. Let $a_{1}, a_{2}, \ldots, a_{n}$ be positive integers (it is allowed that $a_{i}=a_{j}$ for some $i \neq j$ ). Define

$$
N_{d}=\left\{\lambda=\left(\lambda_{1}, \ldots, \lambda_{n}\right) \in \mathbb{N}_{\geq 0}^{n}: \lambda_{1} a_{1}+\lambda_{2} a_{2}+\cdots+\lambda_{n} a_{n}=d\right\} .
$$

By $N_{d}+N_{e}$, we mean the set $\left\{\lambda+\mu: \lambda \in N_{d}, \mu \in N_{e}\right\}$. Thus $2 N_{d}=N_{d}+N_{d}$. Similarly $l N_{d}=N_{d}+\cdots+N_{d}$ (the sum of $l$ copies of $N_{d}$ ). Recall a result in KPS] which will be used in the proof of Lemma 1.4.

Lemma 2.1 ([KPS, Lemma 2.1]). Define $m=\prod_{1 \leq i \leq n} a_{i}$. Then $N_{d m}=d N_{m}$.

Proof of Lemma 1.4. Recall that $m=\prod_{i}^{\prime} a_{i}$ is the restricted product of $a_{1}, \ldots, a_{n}$.

Write $\left\{a_{1}, \ldots, a_{n}\right\}=\left\{b_{1}, \ldots, b_{e}\right\}$, where $b_{i} \neq b_{j}$ if $i \neq j$. We may arrange $a_{1}, \ldots, a_{n}$ so that $a_{1} \leq a_{2} \leq \cdots \leq a_{n}$ and $b_{1}<b_{2}<\cdots<b_{e}$. Moreover, find integers $l(1), \ldots, l(e)$ such that $1 \leq l(1)<l(2)<\cdots<l(e)=n$ and $a_{1}=a_{2}=$ $\cdots=a_{l(1)}=b_{1}, a_{l(1)+1}=a_{l(1)+2}=\cdots=a_{l(2)}=b_{2}, \ldots, a_{l(e-1)+1}=a_{l(e-1)+2}=$ $\cdots=a_{l(e)}=b_{e}$.

In order to prove that $k\left[A_{m}\right]=A_{0} \oplus A_{m} \oplus \cdots \oplus A_{d m} \oplus \cdots$, we will prove by induction that $A_{d m}=A_{m}^{d}$, where $A_{m}^{d}$ is the $k$-vector space generated by elements of the form $h_{1} h_{2} \cdots h_{d}$ with each $h_{j} \in A_{m}$ for $1 \leq j \leq d$.

Each element in $A_{d m}$ is a linear combination of monomials of the form $f_{1}^{\lambda_{1}} f_{2}^{\lambda_{2}} \ldots$ $f_{n}^{\lambda_{n}}$, where $\lambda_{1} a_{1}+\cdots+\lambda_{n} a_{n}=d m$ (note that $f_{j} \in A_{a_{j}}$ for $1 \leq j \leq n$ ). It suffices to show that these monomials $f_{1}^{\lambda_{1}} \cdots f_{n}^{\lambda_{n}} \in A_{m}^{d}$.

For a monomial $f=f_{1}^{\lambda_{1}} f_{2}^{\lambda_{2}} \cdots f_{n}^{\lambda_{n}}$ with $\lambda_{1} a_{1}+\cdots+\lambda_{n} a_{n}=d m$, define $\lambda=$ $\left(\lambda_{1}, \ldots, \lambda_{n}\right) \in \mathbb{N}_{\geq 0}^{n}$. Define $\mu=\left(\mu_{1}, \ldots, \mu_{e}\right) \in \mathbb{N}_{\geq 0}^{e}$ by

$$
\mu_{i}=\sum_{l(i-1)+1 \leq j \leq l(i)} \lambda_{j}
$$

for $1 \leq i \leq e$, where it is understood that $l(0)=0$.

Define

$$
N_{d}^{\prime}=\left\{\eta=\left(\eta_{1}, \ldots, \eta_{e}\right) \in \mathbb{N}_{\geq 0}^{e}: \eta_{1} b_{1}+\cdots+\eta_{e} b_{e}=d\right\} .
$$


It follows that $\mu \in N_{d m}^{\prime}$. Apply Lemma 2.1 to $N_{d m}^{\prime}$. We find there $\mu=\nu^{(1)}+$ $\nu^{(2)}+\cdots+\nu^{(d)}$ for some $\nu^{(1)}, \ldots, \nu^{(d)} \in N_{m}^{\prime}$.

Write $\nu=\nu^{(1)}=\left(\nu_{1}, \nu_{2}, \ldots, \nu_{e}\right)$. It follows that $\mu_{i} \geq \nu_{i}$ for $1 \leq i \leq e$. By Formula (11), we find that $\sum_{l(i-1)+1 \leq j \leq l(i)} \lambda_{j} \geq \nu_{i}$ for $1 \leq i \leq e$. Hence it is possible to find $\lambda_{1}^{\prime}, \ldots, \lambda_{n}^{\prime} \in \mathbb{N}_{\geq 0}$ satisfying the conditions (i) $\lambda_{j}^{\prime} \leq \lambda_{j}$ for $1 \leq j \leq n$, and (ii) $\nu_{i}=\sum_{l(i-1)+1 \leq j \leq l(i)} \lambda_{j}^{\prime}$ for $1 \leq i \leq e$.

Define $g \in A_{m}$ and $h \in A_{(d-1) m}$ by

$$
g=\prod_{1 \leq j \leq n} f_{j}^{\lambda_{j}^{\prime}}, \quad h=\prod_{1 \leq j \leq n} f_{j}^{\lambda_{j}-\lambda_{j}^{\prime}} .
$$

We find that $f=g \cdot h$. Since $h \in A_{(d-1) m}=A_{m}^{d-1}$ by induction, we find that $f \in A_{m}^{d}$.

Before proving Theorem 1.2, we recall a theorem about Noether's bound due to Fleischmann and Fogarty independently.

Theorem 2.2 (Fleischmann, Fogarty $[\mathrm{Fl}],[\mathrm{Fo})$. Let $G$ be a finite group, $k$ be a field, and $V$ be an $r$-dimensional vector space over $k$. Suppose that $G \rightarrow G L(V)$ is a representation of $G$. Let $x_{1}, \ldots, x_{r}$ be a basis of the dual space $V^{*}$ of $V$ such that $k[V]^{G}$ is a $k$-subalgebra of the graded algebra $k[V]=k\left[x_{1}, \ldots, x_{r}\right]$, where each $x_{i}$ is of degree one.

Assume that either char $k=0$ or char $k=p>0$ with $p \nmid|G|$. Then $k[V]^{G}$ is generated over $k$ by invariant polynomials of degree less than or equal to $|G|$.

Proof of Theorem 1.2. Apply Theorem 2.2 to get a set of generators of $A=k[V]^{G}$ and then use Lemma 1.4 .

If there is a non-zero invariant homogeneous polynomial of degree $d$ for all $1 \leq$ $d \leq|G|$, then apply Theorem 2.2 and Lemma 1.4. Note that the integer $m$ in Lemma 1.4 is $\prod_{1 \leq d \leq r} d=|G|$ !. Hence we get $k\left[A_{m}\right]=A_{0} \oplus A_{m} \oplus \cdots \oplus A_{d m} \oplus \cdots$.

Suppose that there is no non-zero invariant homogeneous polynomial of degree $d$ for some $d$ with $1 \leq d \leq|G|$. We just throw away these integers $d$ and proceed as above. Thus we get an integer $m^{\prime}$ such that $k\left[A_{m^{\prime}}\right]=A_{0} \oplus A_{m^{\prime}} \oplus \cdots \oplus A_{d m^{\prime}} \oplus \cdots$. Since $|G|$ ! is a multiple of $m^{\prime}$, it is easy to see that $k\left[A_{m}\right]=A_{0} \oplus A_{m} \oplus \cdots \oplus A_{d m} \oplus \cdots$ if we define $m=|G|$ !.

The remaining proof is the same as the proof of Theorem 3.1 in [KPS].

\section{REFERENCES}

[Fl] P. Fleischmann, The Noether bound in invariant theory of finite groups, Adv. Math. 156 (2000), 23-32. MR.1800251 (2001k:13009)

[Fo] J. Fogarty, On Noether's bound for polynomial invariants of a finite group, Electron. Res. Announc. Amer. Math. Soc. 7 (2001), 5-7. MR1826990(2002a:13002)

[Ha] R. Hartshorne, Algebraic geometry, Springer Graduate Texts in Mathematics, vol. 52, Springer-Verlag, New York, 1977. MR0463157 (57:3116)

[KKV] F. Knop, H. Kraft and T. Vust, The Picard group of a G-variety, in "Algebraic transformation groups and invariant theory", edited by H. Kraft, P. Slodowy and T. A. Springer, DMV Seminar, Band 13, Birkhäuser, Basel, 1989. MR1044586

[KP] S. S. Kannan and S. K. Pattanayak, Projective normality of Weyl group quotients, arXiv: $1007.0606 \mathrm{v} 2$.

[KPS] S. S. Kannan, S. K. Pattanayak and P. Sardar, Projective normality of finite group quotients, Proc. Amer. Math. Soc. 137 (2009), 863-867. MR2457424 (2010c:14050) 
[La] S. Lang, Introduction to algebraic geometry, Interscience Publ., New York, 1958. MR0100591(20:7021)

[Za] O. Zariski, Complete linear systems on normal varieties and a generalization of a lemma of Enriques-Severi, Ann. of Math. (2) 55 (1952), 552-592. MR0048857 (14:80d)

Department of Mathematics, National Taiwan University, Taipei, Taiwan

E-mail address: hchu@math.ntu.edu.tw

Department of Mathematics, Tamkang University, Taipei, Taiwan

E-mail address: sjhu@math.tku.edu.tw

Department of Mathematics and Taida Institute of Mathematical Sciences, National TAiWAn University, TAIPEI, TAIWAN

E-mail address: kang@math.ntu.edu.tw 